\title{
Chanter les territoires Sámi dans un monde plus-qu'humain
}

\author{
Par Stéphane Aubinet
}

Stéphane Aubinet, Doctorant - University of Oslo (Norvège), Department of Musicology.stephaneaubinet@hotmail.com

\section{- Introduction}

Au cours des dernières décennies, l'élaboration de cadres théoriques faisant l'impasse sur le clivage nature-culture a fait l'objet d'un intérêt grandissant. À cheval entre les sciences humaines et naturelles, la géographie n'a pas manqué de suivre cette direction. Les remises en question les plus profondes du clivage ne viennent pas tant de la géographie environnementale, attachée à l'étude des interactions entre le substrat physique de la terre et les comportements humains (Moseley et al., 2014), que d'initiatives cherchant plus radicalement à réintégrer les humains à de plus larges collectifs d'acteurs aux trajectoires entrecroisées. Si des exemples lointains peuvent venir à l'esprit, notamment les travaux d'Élisée Reclus en géographie anarchiste (Clark et Martin, 2004), c'est surtout l'essor des géographies animales, de la théorie de l'acteur-réseau et de la perspective de l'habiter (dwelling perspective) qui retiennent notre attention (pour une synthèse bibliographique de ces approches, cf. Johnston, 2008).

La musicologie, plus fermement ancrée dans le milieu des sciences sociales et des humanités, ne s'est engagée que récemment dans cette voie, notamment à travers une rencontre prometteuse avec le multinaturalisme d'Eduardo Viveiros de Castro (cf. de Mori et Seeger, 2013 ; Gautier, 2016). Cet article se veut une contribution à cet effort épistémologique. Il jette les bases d'une rencontre à trois termes - géographie, musicologie et ouverture aux nonhumains ${ }^{1}$ - articulée autour du concept de monde plus-qu'humain. Celui-ci est développé par le philosophe David Abram dans son ouvrage The Spell of the Sensuous (cf. Abram, 2013 pour une traduction française) afin de mettre en lumière l'appartenance de la perception et du langage humains à un monde où cohabitent animaux, plantes, paysages et air en tant que forces intelligentes. La terre n'y est pas envisagée comme un substrat inanimé et

1. L'expression non-humain renvoie aux animaux, paysages, objets, technologies, esprits et à tout autre type d'êtres avec lesquels les humains entretiennent des relations au sein d'un collectif. 
indifférent à nos pensées auquel la science seule aurait un accès direct, mais comme le lieu même de nos pensées, une entité vivante et respirante dans laquelle nous sommes immergés.

Abram développe ainsi une intuition partagée avec l'anthropologue britannique Tim Ingold selon laquelle les humains ne vivent pas à l'intérieur de leurs corps, mais au sein d'un environnement, entendu non pas comme «ce qui nous entoure », mais comme « une zone d'interpénétration à l'intérieur de laquelle nos vies et celles des autres s'entremêlent en un ensemble homogène » (Ingold, 2013, p. 10). Pour Ingold, c'est dans ce monde unique, fait de matériaux, forces et lignes plutôt que d'intentionnalités situées et contenues en elles-mêmes, que les organismes humains et non humains, continuellement engagés dans leur tâche d'habiter le monde, se constituent un territoire (Ingold, 2000) - une approche qui doit beaucoup à la phénoménologie de Martin Heidegger et Maurice Merleau-Ponty, aux rhizomes de Gilles Deleuze et Félix Guattari et à l'écologie de la perception de James J. Gibson.

Cette rencontre est empiriquement ancrée dans une description de la pratique du joik, répertoire vocal chanté par les Sámi de l'Arctique européen. Elle vise à chercher en quoi cette pratique, d'une part, émane d'un monde fondamentalement plus-qu'humain actualisé à travers différentes modalités de l'habiter et, d'autre part, est impliquée dans la tâche même d'habiter le monde et d'y créer des territoires, notamment en permettant aux interprètes de s'engager dans un environnement où s'entremêlent des processus d'itinérance et d'attention. Deux directions de recherche qui constituent des gestes d'ouverture vers un champ d'étude à construire, et non son programme constitué 2 .

Les indigenous studies ont mis en lumière le danger qui existe à mettre au service d'une science qui ne profite in fine qu'aux chercheurs les communautés indigènes en tant qu'objets d'étude (Smith, 1999). Les Sámi sont particulièrement conscients de cette problématique et il est utile de préciser que l'ambition épistémologique de cet article va de pair avec une volonté de décrire les pratiques musicales Sámi dans leurs propres termes. Il existe, en effet, ce qu'on pourrait appeler une «musicologie indigène Sámi » qui ne rentre pas dans les dichotomies que les chercheurs lui ont appliquées depuis le XIX ${ }^{\mathrm{e}}$ siècle dans leurs analyses : nature et culture, sujet et objet, signifiant et signifié, esprit et corps. Elle attribue notamment l'origine du

\footnotetext{
2. Ce projet se distingue nettement de ceux de l'écomusicologie, puisque les domaines de la musique, de la nature et de la culture ne sont pas sollicités a priori en tant que catégories ontologiques (cf. Gautier, 2016 pour un examen critique de la discipline), et de la zoomusicologie, puisque celle-ci repose sur la frontière entre organisme intentionnel et environnement que nous cherchons à dissoudre et à laquelle elle doit les accusations d'anthropomorphisme qui entravent son développement (cf. Despret, 2013 pour un examen de cette problématique dans l'éthologie).
} 
chant à la terre et aux esprits souterrains, une idée systématiquement rangée par les travaux musicologiques dans les domaines inoffensifs du folklore, de la croyance ou de l'anecdote. Le recours à des ponts conceptuels, tels que le monde plus-qu'humain et l'attention, vise à engager la pensée avec cette « musicologie Sámi » et, ce faisant, à suivre le projet de décolonisation permanente de la pensée proposé par Eduardo Viveiros de Castro (2014, p. 4) et l'invitation au dialogue exprimée par la chanteuse Mari Boine :

«Vous [Les Occidentaux] nous avez fait beaucoup de mal, mais nous sommes prêts à partager avec vous notre perspicacité culturelle et notre sagesse - si vous vous engagez à nous écouter » (Cité par Jones-Bamman, 1993, p. 373, traduction de l'auteur).

La première section de cet article introduit la pratique dite « traditionnelle » du joik et la manière dont il permet aux interprètes de voyager à la rencontre du terrain et de guider leur attention vers les êtres humains et non-humains qui l'habitent. Une deuxième section s'intéresse à l'idée selon laquelle le joik n'aurait sa place que dans la montagne, mettant en lumière l'impact de la colonisation sur les lieux d'interprétation du joik et l'attachement du chant aux paysages fennoscandiens. Les pratiques de trois artistes impliqués dans le joik dit «moderne » (selon l'expression de Jones-Bamman, 1993) - Mari Boine, Wimme Saari et Ánde Somby - sont ensuite abordées afin d'ouvrir une réflexion autour de la place de l'environnement sensoriel et de l'humanité globalisée dans la musique Sámi contemporaine.

\section{- Chanter (dans) le monde sensoriel}

Les Sámi, autrefois appelés « Lapons », habitent un pays situé dans l'extrême Nord de la Fennoscandie. Ils sont héritiers d'un mode de vie semi-nomade lié principalement à l'élevage du renne, toujours pratiqué par une minorité de ses membres. Longtemps victimes d'une politique d'assimilation de la part des états nordiques, la lutte pour la reconnaissance de leurs droits se prolonge jusqu' aujourd'hui avec un relatif succès. La majorité d'entre eux ont aujourd'hui adopté un mode de vie sédentaire et acquis un niveau de vie semblable à celui de leurs voisins nordiques. Ils sont, au sein de l'Union européenne, la seule minorité ethnique à s'être vu attribuer le statut de communauté indigène.

L'identité culturelle revendiquée par les Sámi trouve notamment sa cohérence dans une pratique musicale appelée joik. Sa diversité est telle qu'il est malaisé de tirer des généralités quant à sa forme. On distingue communément trois traditions : le luohti (pratiqué par les Sámi du Nord), le vuollie (pratiqué par les Sámi du Sud) et le leu'dd (pratiqué par les Sámi orientaux, en Russie et dans l'extrême nord-est de la Finlande). C'est plus particulièrement au luohti 
et au vuollie que s'attache cet article. Ceux-ci font un usage parcimonieux de paroles accompagnées de syllabes sans signification, à la différence du leu'dd, qui fait plus fréquemment appel à des textes versifiés et demeure moins étudié par la musicologie. En deçà de leurs différences, le musicologue Paal Fagerheim identifie une série de traits communs :

«Cette musique vocale traditionnelle [...] remplit des fonctions référentielles similaires à travers tout le territoire, évoquant des personnes, objets, lieux, ou tout autre élément extérieur. La pratique vocale du joik a traditionnellement été interprétée sans accompagnement musical. [...] Les interprètes soulignent le fait que les joiks sont circulaires, en ceci qu'ils n'ont pas de début ou de fin clairement définis. L'interprète peut dès lors changer de point de départ à chaque fois qu'un joik est interprété. De fait, un joik est considéré comme ayant une qualité existentielle assez différente d'autres types "d'objets" musicaux. Le joik "existe" en ses propres termes, et l'interprète qui le chante se connecte à lui depuis différents points de départ » (Fagerheim, 2014, p. 65, traduction de l'auteur).

Au sein d'une communauté Sámi, chaque membre se voit attribuer un joik qui lui fait office de portrait musical. On rencontre également des joiks faisant référence à d'autres espèces, tels que le joik du loup ou le joik de l'ours, et des joiks évoquant des éléments de l'environnement, tels que le joik d'une rivière ou celui d'une montagne. Il ne s'agit pas d'un simple procédé de représentation, puisque l'interprétation d'un joik est censée rendre présent ce à quoi il fait référence afin d'invoquer des souvenirs qui lui sont associés. Le chant et ce qu'il désigne sont ainsi ontologiquement liés, si bien que les Sámi insistent sur le fait qu'ils ne chantent pas à propos des animaux, mais qu'ils chantent les animaux.

La question des rapports entre ces chants, fréquemment revendiqués par les Sámi comme la plus ancienne tradition musicale de l'Europe, et la formation des territoires Sámi nécessite que l'on s'entende sur le sens du terme territoire. La littérature ethnologique a coutume de représenter celui des Sámi à la manière d'une surface délimitée sur une carte de la Fennoscandie septentrionale. Celle-ci s'étend de part et d'autre du cercle polaire Arctique, couvrant quelque $400000 \mathrm{~km}^{2}$ répartis sur quatre pays : la Norvège, la Suède, la Finlande et la Russie.

Cette pratique de représentation pose question dès que l'on décide d'adopter le point de vue des premiers concernés. Les éleveurs Sámi, en effet, montrent peu d'intérêt pour les cartes : «Les cartes, c'est pour les touristes !» (Mazzullo et Ingold, 2008, p. 30, traduction de l'auteur). De fait, les savoirs géographiques dits « vernaculaires », particulièrement élaborés au sein des groupes nomades ou héritiers du nomadisme, ne peuvent être que pluriels et se communiquent difficilement sur le mode abstrait des cartes, à l'inverse des savoirs dits « savants », qui sont « discursifs, formalisés sur un mode théorique, soucieux d'objectivation et de communicabilité hors contexte » (Collignon, 2005, p. 324). Les territoires Sámi, de ce point de vue, ne se 
constituent pas de l'extérieur, via un surcodage cartographique de la terre, mais de l'intérieur, via un engagement des acteurs dans leur environnement (Ingold et Kurttila, 2000 ; Helander-Renvall, 2008).

Ainsi, un lieu tel qu'une montagne ne constitue pas, pour l'éleveur Sámi, un point localisable dans l'espace par des coordonnées géographiques. Un lieu est avant toute chose une étape le long d'un itinéraire prenant sens dans le cadre d'une activité (Mazzullo et Ingold, 2008). L'espace habité (báiki) doit être rendu vivant à travers l'itinérance et le montage de la tente conique (lavvú), à l'intérieur de laquelle un feu est ensuite allumé et le café préparé - du moins dans les milieux où l'élevage semi-nomade est encore pratiqué (Ingold et Kurttila, 2000). Il en va de même pour les territoires régionaux (guovlu) partagés par une communauté, qui ne prennent sens qu'à travers une familiarité avec ses lieux et le souvenir des ancêtres qui les ont parcourus par le passé, et pour des lieux plus précisément localisés (sadji), identifiés et nommés d'après l'activité qui est menée (e.g. buoððosadji, un lieu pour capturer les saumons au filet) (Helander, 1999). L'espace habité par les Sámi n'est pas donné d'avance : il doit être actualisé par l'itinérance, l'engagement dans des activités concrètes qui y sont menées et leur remémoration pour émerger à la vie.

Ces éléments doivent être gardés à l'esprit lorsqu'on s'intéresse aux joiks faisant référence à des éléments du terrain, ce que semblent avoir négligé de faire les chercheurs qui s'y sont intéressés au joik. Le musicologue Ola Graff décrit ainsi l'acte de chanter une montagne par analogie avec celui de la nommer : il s'agirait d'extraire la montagne de l'espace sauvage afin de l'inclure dans l'univers mental de l'interprète (Graff, 2007). Une manière, en somme, de domestiquer le terrain sauvage en y appliquant le sceau de la culture - un leitmotiv de l'analyse anthropologique du $\mathrm{XX}^{\mathrm{e}}$ siècle.

Or, comme l'ont montré des travaux récents, la distinction entre espaces domestiques et espaces sauvages ne trouve sa pleine pertinence qu' au sein du monde moderne occidental (pour un examen approfondi de la question, cf. Descola, 2005). Chez les Sámi, en particulier, une vaste documentation ethnographique atteste le fait que la nature n'est a priori ni étrange ni incompréhensible ; elle constitue un complexe de sentiers liés à l'exploitation de ressources naturelles et désignés chez les Sámi du Nord par le terme meahcci. Bien que situé à l'écart des lieux habités (báiki), meahcci est vécu comme un espace familier ; on ne peut pas s'y perdre, car on y est «à la maison » (Schanche, 2002).

En outre, comme l'anthropologue Philippe Descola s'attache à le mettre en évidence, l'idée même que les territoires se forment à travers un processus d'appropriation collective de la terre semble être le fruit de la trajectoire 
historique singulière du monde occidental et ne peut rendre compte du rapport au territoire dans les collectifs non-modernes (Descola, 2016, leçon du 2 mars 2016).

Il semble donc trompeur de décrire la fonction du joik en ces termes, et c'est en effet dans une tout autre direction que les interprètes nous emmènent lorsqu'ils décrivent leur expérience :

«Si je veux chanter, seul ou avec une ou deux autres personnes, un lieu particulier dans la nature, comme un torrent ou une montagne remarquable, j'effectue un voyage vers ce lieu en pensée. On se rend jusque là-bas, on voit l'endroit par soi-même, et d'une certaine manière, on est là. C'est une sorte de voyage que l'on fait par le chant. On ressent une affinité avec ce lieu » (Ánte Mikkel Gaup, cité dans Eriksson, 2002: 131-132, traduction de l'auteur).

Chanter revient à voyager à la rencontre du terrain, à parcourir les sentiers, les rencontres et les activités qui sont liés à la montagne, si bien qu'en offrant à l'interprète une modalité autre que le mouvement physique pour explorer l'espace, le chant ne se contente pas d'affirmer des attachements préexistants, mais joue une part considérable dans la formation d'une conscience territoriale ${ }^{3}$.

Ce type de voyage dans l'esprit peut également être opéré en citant une série de lieux par leurs noms (Mazzullo et Ingold, 2008), procédé répandu parmi d'autres groupes nomades ou héritiers du nomadisme ${ }^{4}$. La différence est la suivante : «Tu ne peux pas répéter un nom toute la journée, n'est-ce pas ? Mais un joik, tu peux le chanter constamment » (propos d'un chanteur Sámi rapporté par Hanssen, 2011, traduction de l'auteur). Semblable en certains points aux songlines australiennes, le joik s'en distingue notamment par son caractère circulaire plutôt que linéaire (cf. Somby, 2007) : l'ensemble de l'interprétation s'articule autour d'un seul lieu cerné par l'horizon et les joiks de différents lieux reliés par un itinéraire ne sont, en principe, pas chantés en série.

Il ne s'agit pas ici de remettre en question l'analogie proposée par Ola Graff entre l'attribution d'un joik à un paysage et le fait de le nommer analogie que certains interprètes n'ont d'ailleurs pas manqué de relever euxmêmes (Hanssen, 2011) -, mais de s'entendre sur ce qu'implique l'acte de «nommer ». Nous suivons ici la philosophe Isabelle Stengers, pour qui nommer n'implique pas la projection de significations culturelles sur un monde insignifiant en lui-même, mais une opération pragmatique «qui

3. Cette capacité de la musique à agencer l'espace n'est pas inconnue de la musicologie (cf. Weisethaunet, 2007).

4. L'un des exemples les plus fréquemment cités est rapporté par Keith Basso (1988) au sujet des Apache orientaux. 
confère à ce qui est nommé le pouvoir de nous faire sentir et penser sur le mode qu'appelle le nom » $(2009$, p. 13).

Il s'agit avant tout de guider l'attention de l'interprète et des auditeurs afin de rendre présent le lieu chanté. Au début du XX ${ }^{\mathrm{e}}$ siècle, l'ethnologue amateur Karl Tirén prétendait ainsi que le joik imite mélodiquement les reliefs du paysage : un pic montagneux correspondrait, par exemple, à une note élevée dans la mélodie qui lui est associée (Lüderwaldt, 1976). Il semble néanmoins que des exemples aussi simplistes soient des exceptions et que, plus fréquemment, ce soit l'atmosphère générale du lieu que le joik cherche à évoquer par le son, selon des codes musicaux qui ne peuvent être que partiellement assimilés par une oreille extérieure, mais qui sont intuitifs pour toute personne ayant grandi dans le milieu du joik. Dans un cas comme dans l'autre, quelque chose de la montagne est toujours présent dans le chant qui lui correspond. La chanter revient à la montrer du doigt, à rendre sa présence directement perceptible.

Le recours au concept d'attention n'est pas anodin. Il occupe une place centrale dans l'œuvre d'Ingold, pour qui la connaissance de l'environnement ne se transmet pas en communiquant une série d'informations propositionnelles d'une génération à une autre - autrement dit, elle ne circule pas en circuit fermé au sein de réseaux sémiotiques strictement humains -, mais en montrant les choses et, à travers l'intégration de savoir-faire (skills), en ouvrant la perception aux significations immanentes du monde plus-qu'humain, processus qu'il désigne par l'expression d'éducation de l'attention (Ingold, 2000). On pourrait, en français, parler de faire connaissance, dans sa double acception de s'engager dans une rencontre et de créer de la connaissance; chanter pour faire connaissance avec la montagne, et non pour la domestiquer.

Les joiks d'animaux semblent impliquer le même processus. Pour le chanteur Terje Trednes, l'une de leurs fonctions fondamentales est, précisément, de permettre aux enfants de faire connaissance avec l'animal chanté :

«En écoutant le joik du loup, les enfants pouvaient entendre que cet animal pouvait être dangereux. [...] Il y a plusieurs manières de chanter le joik du loup, par exemple quand le loup a mangé du renne et n'a plus besoin de chasser. Il est tranquille, contrairement au loup affamé en train de chasser, qui est agité. Mais tous deux peuvent être chantés, tout comme l'ours. C'était destiné aux enfants, afin qu'ils associent une personnalité à ces animaux » (entretien à Kárášjohka, janvier 2017, traduction de l'auteur).

Comme dans le cas de la montagne, les personnalités véhiculées par le chant du loup ne sont pas des constructions purement culturelles qu'il s'agirait d'imposer à un animal insignifiant en lui-même, mais des intensités perçues à travers un engagement avec l'animal au sein de l'environnement. Il s'agit donc d'une relation de stimulation mutuelle : la perception nourrit le chant et, à travers une éducation de l'attention, le chant nourrit à son tour la perception. 
La traduction, dans le chant, de la personnalité d'un animal passe souvent par l'évocation concrète des sons ou des mouvements de ce dernier : l'ours sera volontiers chanté sur un rythme lent ou avec une voix gutturale, tandis que le corbeau sera chanté sur un rythme sautillant ou avec une voix croassante et le lièvre sur un rythme rapide. La familiarité avec l'animal constitue, dans tous les cas, un prérequis pour pouvoir le chanter.

«Quand j'étais enfant, on épiait le renard, on étudiait sa démarche : il guette le gibier qui lui servira de nourriture, il marche en se balançant un peu, et tout à coup il s'arrête. Alors, on faisait un juoigos [joik] où tout ça s'entendait. C'était le juoigos du renard, et il ressemblait au renard » (propos d'un chanteur Sámi rapporté par Delaporte, 1978, p. 111).

D'une certaine manière, le renard contribue à composer son propre chant. Certes, ce chant diffère largement d'une communauté Sámi à une autre et ce sont les interprètes humains qui feront la démarche concrète de composer le joik et de l'intégrer à leur répertoire. Pour autant, il ne sera jugé valide qu'à condition qu'il évoque l'animal avec succès. Il n'est pas rare, en effet, que les auditeurs, à l'écoute d'un joik nouvellement composé, le rejettent sous prétexte que l'animal ne ressemble pas à la mélodie qu'ils entendent (Arnberg et al., 1997). Indirectement, le renard aura le dernier mot. L'identité du compositeur est d'ailleurs insignifiante et, bien souvent, inconnue : le chant est exclusivement associé qu'à ce qu'il est censé évoquer.

L'engagement du joik dans la constitution de territoires ne se comprend donc qu'en le replaçant dans le monde plus-qu'humain dont il émane. À travers les chants de lieux, d'animaux et de personnes qui constituent leur environnement sensoriel, les interprètes et auditeurs du joik font connaissance avec leurs territoires, non pas en incorporant une carte cognitive dans leurs cerveaux, mais « comme un artisan expérimenté connaît son matériau : en étant attentif à ses formes et textures, en pouvant répondre à ses variations avec créativité et en restant alerte envers les possibilités qu'il offre afin de poursuivre diverses tâches » (Ingold et Kurttila, 2000, p. 186, traduction de l'auteur).

\section{- La montagne comme refuge}

Selon une idée répandue, c'est dans la montagne que le joik a sa place : «C'est là qu'il doit être. Il n'a pas sa place dans la ville » (Ole Larsen Gaino, dans Lundby, 2014, traduction de l'auteur). Il existe au moins deux manières de comprendre cet attachement. D'une part, la tradition attribue aux gufihttar - esprits anthropomorphes vivant sous terre et surveillant la bonne conduite des humains dans la montagne - l'origine du joik, enseigné postérieurement aux humains. Ce serait donc dans l'interaction avec la montagne et ses 
habitants que le joik serait venu aux Sámi, idée développée dans la section suivante de cet article.

D'autre part, la condamnation du joik par les autorités nordiques eut pour conséquence une raréfaction dramatique des interprétations au sein de l'espace public. Dès le XVII ${ }^{\mathrm{e}}$ siècle, l'association de ces chants avec l'ancienne religion Sámi et les cérémonies chamaniques leur valurent l'appellation de «musique du diable »- de fait, s'il ne faisait pour les missionnaires de l'époque aucun doute que le joik venait des entrailles de la Terre, celles-ci n'étaient pas habitées par des gufihttar bienveillants, mais par Satan. Au $\mathrm{XIX}^{\mathrm{e}}$ siècle, tandis que la christianisation était supposée achevée, c'est à l'alcoolisme qu'ils furent associés par le milieu læstadien ${ }^{5}$, car les personnes ivres osaient plus facilement défier le tabou et chanter en public. Enfin, la montée du darwinisme social et les politiques d'intégration menées par les états nordiques véhiculèrent l'idée selon laquelle le joik serait une musique « primitive » destinée à disparaître (Graff, 2016).

Malgré le danger de stigmatisation - voire, dans certains cas, de condamnations - lié à l'interprétation de chants interdits, la tradition put se perpétuer en trouvant refuge dans la montagne, à l'écart des autorités, comme l'exprime le poète Paulus Utsi :

«Quand j'arrive dans la montagne

Je suis chez moi

Là, j'ose élever ma voix dans un joik joyeux

Les contraintes et interdits

étrangers n'atteignent pas cet endroit

Ici, je suis chez moi ${ }^{6}$. »

(Utsi, 2000, p. 236, traduction de l'auteur)

La montagne ne correspond, chez les Sámi, ni à l'espace laid et effrayant décrit par les missionnaires et voyageurs du XVII ${ }^{\mathrm{e}}$ au XIX ${ }^{\mathrm{e}}$ siècle ${ }^{7}$, ni aux espaces sauvages que les Scandinaves amateurs de friluftsliv (« vie à l'air libre ») visitent durant leur temps libre, mais à l'espace familier désigné par le terme meahcci (cf. section précédente). Les règles de comportement y sont à ce point spécifiques que Dieu lui-même semble y adapter son jugement : une même personne pourra condamner sévèrement le joik comme une activité

\footnotetext{
5. Le læstadianisme est un mouvement religieux conservateur trouvant son origine dans la doctrine du pasteur suédois Lars Levi Læstadius (1800-1861).

6. Le terme suédois fjäll (norvégien fjell), traduit ici par «montagne », désigne toute zone située au-delà de la limite des arbres, couvrant ainsi une partie considérable des régions septentrionales du pays Sámi, d'où Paulus Utsi est originaire.

7. Le missionnaire Johannes Schefferus estimait notamment que la superstition des Sámi « vient de ce qu'ils demeurent dans un pays affreux, au milieu des forêts, parmi les bêtes sauvages » (1678, p. 61).
} 
de péché dans le village et chanter sans complexe une fois dans la montagne (entretien avec Terje Trednes à Kárášjohka, janvier 2017).

Considéré dès lors comme un chant d'extérieur, ce n'est qu'avec beaucoup de difficulté que le joik put s'imposer dans les espaces intérieurs des zones habitées (báiki) au cours du XX ${ }^{\mathrm{e}}$ siècle. Sa diffusion sur les chaînes de radio du Finnmark fut vivement critiquée par certains auditeurs qui ne pouvaient supporter d'entendre un joik chanté à pleine voix dans leur salon (Graff, 2016). Il demeure interdit dans plusieurs églises des régions septentrionales du pays Sámi, notamment à Guovdageaidnu, non plus parce que le joik serait intrinsèquement diabolique, mais parce que l'intérieur de l'église est jugé comme un lieu totalement inapproprié à son interprétation (Guttormsen, 2014).

Cette association entre le joik, l'extérieur et la montagne est aujourd'hui remise en question par les pratiques de certains artistes de joik dit « moderne ». L'interprète Johan Anders Bær estime ainsi que le joik peut parfaitement prendre sens dans le paysage d'une ville industrielle, à condition d'adapter sa forme à ce nouvel environnement et de devenir " autre chose » (entretien à Kárášjohka, janvier 2017). On pensera également à la piste Texas de l'artiste Wimme Saari (1996), qui évoque des paysages états-uniens via l'incorporation au joik d'éléments de musique électronique et d'une série de «Oh yeah » dans les paroles. Dans les régions méridionales du pays Sámi, le musicien Frode Fjellheim a permis au joik de faire son entrée à l'église en composant une «messe arctique » (2004) intégrant vuollie (tradition régionale du joik), musiques traditionnelles européennes et textes liturgiques en langue Sámi du Sud. Quelle que soit la démarche sous-jacente, la musique Sámi contemporaine s'est progressivement fait une place à la radio, dans les festivals et salles de concert, dans certaines églises, à la télévision et au cinéma, quittant ses montagnes pour apparaître au grand jour comme jamais auparavant.

Paradoxalement, en se réappropriant de la sorte les paysages sonores de báiki, ces artistes ne font qu' affirmer l'attachement profond du joik aux paysages de meahcci, puisque cette ouverture ne s'effectue que moyennant une transformation du chant et l'incorporation d'éléments appartenant aux nouveaux environnements explorés, qu'il s'agisse de langues ou d'instruments étrangers, de schémas d'organisation formelle ou de techniques d'arrangement, de technologies ou d'esthétiques musicales. Comme le renard, le Texas contribue à la composition de son propre joik en imposant ses termes à la perception de l'interprète et des auditeurs et en gardant la possibilité d'être en désaccord avec le chant composé. 


\section{- Technologies et territoires humains}

C'est à la fin des années 1960 que le joik dit «moderne » fait son apparition, sous l'impulsion des albums pionniers de l'artiste emblématique Nils-Aslak Valkeapää ${ }^{8}$. Un raccourci naïf, encore invoqué par certains chercheurs scandinaves, consiste à qualifier de tradition authentique le joik tel qu'il fut pratiqué avant cette entrée dans le marché de la musique, par opposition aux pratiques hybrides où le joik rencontre le jazz, le rock, l'electro et d'autres répertoires du monde (cf. Gaski, 2008 ; Graff, 2016). C'est pourtant se fourvoyer dans des idées essentialistes bien étrangères à la pratique du joik, qui semble impliquer une ouverture sur le monde et un sens de l'accueil plutôt qu'un repli identitaire.

De fait, la notion d'authenticité, centrale dans le discours musical du $\mathrm{XX}^{\mathrm{e}}$ siècle bien au-delà du contexte Sámi (Weisethaunet, 2010), inclut dans le cas du joik une idée de pureté. Pour être authentique, «il faut que la musique appartienne "totalement" à la culture dont elle est issue » (da Lage, 2008, p. 20). Or, en ignorant même l'influence prolongée des répertoires musicaux scandinaves dans certaines régions du pays Sámi - audibles dans des enregistrements largement antécédents à l'essor du joik dit « moderne » -, prétendre que le joik dit « traditionnel » n'appartient qu'à la culture dont il est issu revient à négliger les échanges, plus riches encore, entretenus entre les Sámi et le monde plus-qu'humain au sein duquel ils vivent. Or, ces échanges semblent être à la base même du joik, comme le suggère l'interprète Torgeir Vassvik :

«Ce qui est spécial avec le joik, c'est qu'il est incroyablement ancien. On n'avait pas d'instrument autre que la voix, excepté le tambour [chamanique], comme tu le sais. C'est la nature qui a été l'instrument du joik. On chantait avec la nature et on imitait la nature. Les sons de la nature, dans toute leur diversité, ont été l'instrument du joik. Ça a influencé le joik, la manière d'utiliser la voix et d'autres choses. Ça a toujours été un dialogue » (Entretien à Oslo, octobre 2016, traduction de l'auteur).

À l'inverse, accepter ce dialogue avec la nature sous prétexte que seuls les échanges avec l'Occident moderne sont assez nocifs pour compromettre l'authenticité des répertoires traditionnels (cf. da Lage, 2008) relèverait de l'idée du « Grand Partage » (Latour, 1991), selon laquelle les modernes se seraient extirpés des traditions, des croyances et de l'emprise de la nature dont les cultures non occidentales sont captives - idée dont le caractère problématique n'est plus à prouver.

\footnotetext{
8. Cet essor est contemporain de protestations de plus en plus médiatisées contre les politiques d'assimilation culturelle adoptées par les états nordiques, qui atteignirent leur climax en Norvège lors de la « controverse d'Alta », liée à la construction d'un barrage dans des zones de pâturage de rennes au tournant des années 1980 .
} 
Il demeure néanmoins incontestable qu'une transition vers des territoires jusqu' alors inexplorés est à l'œuvre dans le joik dit «moderne ». La mondialisation, écrit le géographe Denis Retaillé, ne réalise pas la fin des territoires : « D'autres territoires s'installent, auxquels il est nécessaire de donner d'autres références épistémologiques que la continuité topographique » (Retaillé, 2005, p. 178). Ainsi, dans le joik dit «moderne », le lieu d'interprétation se trouve, pour la première fois, détaché du lieu d'écoute ; une série d'intermédiaires liés aux technologies modernes se sont intercalés entre ces deux moments de l'activité musicale. Cette rupture ne consacre pas pour autant la déterritorialisation absolue du joik, mais un nouvel engagement au sein de territoires identitaires largement détachés de l'environnement sensoriel : tandis que le joik dit « traditionnel » s'est développé au sein d'attachements avec les paysages, animaux et esprits avec lesquels les interprètes cohabitent, nombreux sont les artistes de musique Sámi qui se positionnent désormais au sein de l'humanité globalisée. On se définira, par exemple, comme Sámi ou comme indigène, par opposition aux Scandinaves ou à l'Occident.

Ainsi, chez la chanteuse Mari Boine, figure majeure de la world music, le joik ne se développe pas tant le long d'itinéraires tissés à travers le terrain que dans un monde globalisé où les négociations avec le monde occidental et la critique de leurs politiques de destruction de la nature sont devenues une priorité nouvelle. Il s'agit d'un thème récurrent au sein du joik dit «moderne », illustré par les paroles de la chanson Gula Gula:

« Entends la voix des ancêtres, entends.

Elles te demandent pourquoi tu as laissé la terre devenir polluée, empoisonnée, épuisée.

Elles te rappellent d'où tu viens, entends-tu ?

À nouveau, elles te rappellent que la terre est notre mère.

Si nous prenons sa vie, nous mourrons avec elle. »

(Boine 1989, traduction de l'auteur)

Le recours à des instruments de musique issus de minorités ethniques de diverses régions de la planète contribue à affirmer l'attachement des Sámi à la diaspora indigène globale. La voix de Boine elle-même a souvent été décrite comme sonnant « amérindienne » plutôt que Sámi (Thomassen, 2010). On peut également voir dans Gula Gula un processus d'éducation sensorielle envers les traits qui connectent les Sámi au monde indigène. Ces traits existent indubitablement, mais ils ont besoin d'être actualisés, d'être portés à l'attention. Le philologue Harald Gaski rapporte ainsi comment, lors du World Council of Indigenous Peoples à Port Alberni (Canada) en 1975, l'assemblée doutait fortement du caractère indigène des Sámi jusqu'à ce que Nils-Aslak Valkeapää dissipe instantanément leur scepticisme en chantant un joik (Gaski, 2008). La construction d'une identité indigène dans la musique de Mari Boine ne se fait pas ex nihilo, mais en favorisant la perception 
de certaines intensités du joik et des répertoires indigènes lointains jugées pertinentes, déployant ainsi un nouveau territoire à explorer.

Chez Wimme Saari, le joik est utilisé pour chanter un attachement avec des humains qu'il n'a vraisemblablement rencontrés qu'à travers les médias modernes, tels que Bill Clinton, George Bush et Yasser Arafat (Diamond, 2011). D'après David Abram, l'émergence de ces médias modernes a fortement accentué l'abstraction vis-à-vis de nos rencontres sensorielles directes, déjà entamée par l'adoption de l'écriture. En rendant possible des rencontres incorporelles avec des humains vivant à l'autre bout de la planète, ils canalisent nos sens dans les réseaux d'une « communication désormais strictement humaine » (Abram, 2013, p. 328). L'acte de chanter des figures politiques internationales prend ainsi place au sein d'un mode particulier de relation aux technologies, à l'humanité et à la terre.

Partant de constats similaires, Ola Graff décrit la situation de la manière suivante : « le joik ne vit plus seulement au sein de petites sociétés locales, mais est utilisé en relation avec une grande société Sámi, une grande société norvégienne et une grande société mondiale » (2016, p. 182, traduction de l'auteur). Or, si l'on prend le parti de considérer le territoire comme un champ de relations dont émane la production musicale plutôt qu'un espace inerte occupé par des humains, il s'avère que le passage du joik au global chez Mari Boine ou Wimme Saari ne se fait qu'au prix de l'exclusion de certains attachements au monde plus-qu'humain, si bien que le territoire indigène global que crée un album comme Gula gula est à la fois plus étendu que celui du joik dit « traditionnel » et plus restreint.

Ainsi, l'argument d'Ola Graff, sans être entièrement erroné, présente deux erreurs de langage puisque, d'une part, les territoires du joik dit « traditionnel » ne sont pas plus petits que ceux du joik dit «moderne », et d'autre part, il n'est pas tant question de sociétés que de collectifs rassemblant en leur sein animaux, paysages et technologies. Plus qu'une différence d'échelle, ce sont différentes modalités d'habiter et d'être attentif au monde qui sont exprimées dans les pratiques contemporaines.

On notera toutefois que ces deux modes d'attention ne sont pas mutuellement exclusifs. La plupart des artistes du joik dit «moderne »sont également des interprètes «traditionnels » respectés, comme c'est le cas pour Wimme Saari. En outre, l'association entre technologies musicales modernes et détachement du monde sensoriel n'est pas systématique. C'est ce dont atteste l'album Yoiking with the winged ones (2016) de l'interprète Ánde Somby, réalisé en collaboration avec l'acousticien Chris Watson. L'idée des deux collaborateurs était d'enregistrer les joiks d'Ánde Somby à l'extérieur, en rendant audible l'environnement sonore dans lequel le chant s'inscrit. Parmi 
les joiks interprétés figure celui des esprits souterrains (Gufihttar). Il fut enregistré dans les îles Lofoten (Norvège), archipel dont le relief montagneux est idéal pour travailler sur les échos :

« Nous avons fait ça en juin, nous avions donc beaucoup d'oiseaux répondant au joik, et aussi des poissons interagissant. C'était très fascinant. [...] Le joik a été donné aux Sámi par les êtres souterrains. En d'autres termes, la terre nous a donné le joik. Et donc, pour moi, travailler avec ces échos signifie que je peux communiquer avec ces êtres souterrains » (Ánde Somby, entretien à Troms $\varnothing$, janvier 2015, traduction de l'auteur).

La piste Gufihttar est le fruit d'une rencontre sensorielle entre humains, oiseaux, poissons, vallées et êtres souterrains interagissant dans un même champ sémiotique ${ }^{9}$. Il ne s'agit pas ici de déplacer la pratique musicale vers des réseaux d'appartenance globaux, mais au contraire d'explorer le rapport entre la voix et le terrain, de s'engager dans le monde phénoménal en tentant le pari d'une conversation musicale avec l'environnement sensoriel - ce qui exprime concrètement l'idée selon laquelle les non-humains participent à la composition musicale.

Ainsi, l'attachement des humains à la terre, exploré de manière extensive par Mari Boine en invoquant la figure d'une terre-mère commune à l'humanité, est exploré par Ánde Somby de manière intensive : la notion même de terre se trouve dissoute à mesure que se déploie la multiplicité des êtres qui la constituent localement. Ce n'est qu'en passant par des technologies avancées et le savoir-faire de Chris Watson que cette animation de la terre a pu être rendue dans toute sa profondeur.

L'assimilation par les auditeurs occidentaux est facilitée, d'une part, par l'emploi de termes issus du folklore européen - fairies («fées »), elves (« elfes ») et winged ones ( «êtres ailés ») - afin de désigner les Gufihttar, et d'autre part, par le choix des îles Lofoten, qui passent difficilement pour un lieu dont émane la culture Sámi étant donné son occupation prolongée par des populations scandinaves sédentaires. L'album échappe ainsi à un certain exotisme en déplorant une guerre générale aux êtres magiques qui habitent la terre (Watson, 2016). Comme c'était le cas pour le joik dit «traditionnel » et la musique de Mari Boine, on peut voir dans l'écoute de Yoiking with the winged ones une éducation de l'attention, dirigée ici vers la vie qui anime l'environnement de l'auditeur et la voix des animaux, des paysages et des lutins qui s'y font entendre.

\footnotetext{
9. On notera à ce propos l'absence de paroles et le caractère a priori non-symbolique de l'interprétation - rendant cette dernière potentiellement signifiante pour des agents non-humains (pour une analyse des rapports entre sémiotiques humaines et animales, cf. Kohn, 2013). De nombreux chanteurs Sámi, tels qu'Ánde Somby (entretien à Troms $\varnothing$, janvier 2015), Torgeir Vassvik (entretien à Oslo, octobre 2016) ou Ánte Mikkel Gaup (Eriksson, 2002), affirment d'ailleurs avoir pu communiquer avec les animaux grâce au joik.
} 


\section{- Conclusions}

Si les pratiques musicales peuvent être décrites en tant que «marqueurs d'appartenance des individus et de l'espace» (Canova, 2014, p. 9), leur implication dans la constitution même de l'espace et de ces appartenances doit être gardée à l'esprit, car le territoire ne préexiste pas à ceux qui le chantent : que l'on ait affaire à un éleveur chantant l'ours sur les plateaux du Finnmark, à un duo d'artistes enregistrant un joik dans les îles Lofoten ou à une star de la world music chantant sur scène son attachement au monde indigène, c'est un même processus de mouvement et d'éducation de l'attention qui aboutit à l'actualisation de multiples territoires plus ou moins ancrés dans le monde sensoriel.

Prétendre que le chant façonne les territoires (et inversement) n'implique nullement un dépassement de la fracture nature-culture. Un tel argument pourrait tout aussi bien consolider les deux domaines moyennant une relation dialogique entre les deux, à la manière de l'écomusicologie ou de la géographie environnementale. En revanche, ils perdent leur poids ontologique dès qu'ils sont considérés comme deux modes d'actualisation d'un monde fondamentalement plus-qu'humain. On retient ainsi pour l'analyse musicologique deux démarches également mises en œuvre par Ánde Somby dans Yoiking with the winged ones : d'une part, prendre au sérieux l'idée selon laquelle la terre a enseigné le chant aux humains, et d'autre part, déployer, par l'attention, la multiplicité des présences humaines et non humaines recouvertes par les notions de terre et de territoire.

En ce sens, les montagnes, les villes, les animaux, l'église, le Texas, les technologies médiatiques et les êtres souterrains ne sont pas considérés comme les figurants d'un catalogue « déjà là » dans lequel le génie musical humain puiserait son inspiration, mais comme autant d'êtres activement engagés dans la créativité musicale ; une toile de relations attentionnelles dont émanent différentes formes de chant et les territoires qui leur correspondent. Par le joik, la terre est actualisée, parcourue, explorée ; elle devient « encore plus vivante qu' on ne le croyait » (Helander-Renvall 2008, p. 48, traduction de l'auteur). Cette conception du chant semble jouir d'une solide base phénoménologique puisqu'insuffler de la vie au monde plus-qu'humain renvoie à l'expérience concrète de la respiration et de la voix :

«C'est [...] de par le pouvoir de ce souffle continuel que la nature se renouvelle sans cesse. Le monde autour de nous est une énonciation continue, perpétuelle! Ainsi, l'acte de parler, comme celui de respirer, ne lie pas seulement les humains à Dieu, mais à tout ce qui nous entoure, des pierres aux moineaux » (Abram, 2013, p. 330).

Si l'on suit l'intuition d'Abram, l'étude des pratiques musicales a un rôle central à jouer dans la remise en question de la fracture moderne entre les 
humains et le monde et l'on ne peut qu'encourager la géographie musicale à s'engager dans cette voie.

\section{- Méthodologie}

Entrepris dans le cadre d'un mémoire de master à l'Université catholique de Louvain (Belgique) puis d'un doctorat à l'Université d'Oslo (Norvège), le travail de recherche à l'origine de cet article est basé sur (1) un examen de la littérature sur le joik, principalement issue du milieu académique scandinave, (2) une série d'entretiens effectués en Norvège entre avril 2014 et janvier 2017 avec des musiciens, auditeurs et chercheurs impliqués dans le milieu du joik et (3) l'apprentissage de la pratique du joik auprès d'interprètes Sámi.

\section{Bibliographie}

Abram D. (2013), Comment la terre s'est tue. Traduit de l'anglais par Didier Demorcy et Isabelle Stengers. Paris : La Découverte.

Arnberg M., Ruong I. et Unsgaard H. (1997), Jojk: En presentasjon av samisk folkmusik. Stockholm : Sveriges Radio.

Basso, K. H. (1988), " "Speaking with Names": Language and Landscape among the Western Apache », Cultural Anthropology, vol. 3, no 2, p. 99-130.

Canova N. (2014), La musique au cœur de l'analyse géographique. Paris: L'Harmattan.

Clark J. P. et Martin C. (2004), Anarchy, Geography, Modernity: The Radical Social Thought of Élisée Reclus. 0xford : Lexington.

Collignon B. (2005), "Que sait-on des savoirs géographiques vernaculaires ? », Bulletin de l'Association des géographes français, vol. 82, no 3, p. 321-331.

Da Lage É. (2008), « Politiques de l'authenticité », Volume !, vol. 6, no 1, p. 17-32.

De Mori B. B. et Seeger A. (2013) «Introduction: Considering Music, Humans, and Nonhumans », Ethnomusicology Forum, vol. 22, no 3, p. 269-286.

Delaporte Y. (1978), "Aspects psycho-sociologiques du chant lapon », Yearbook of the International Folk Music Council, vol. 10, p. 109-116.

Descola Ph. (2005), Par-delà nature et culture. Paris : Gallimard.

Descola Ph. (2016) Les usages de la terre. Cosmopolitiques de la territorialité. Cours donné au Collège de France, en ligne :

http://www.college-de-france.fr/site/philippe-descola/course-2015-2016.htm

Despret V. (2013), «From secret agents to interagency », History and Theory, vol. 52, p. 29-44.

Diamond B. (2011), "The Music of Modern Indigeneity. From identity to alliance studies », in Dan Lundberg et Gunnar Ternhag. Yoik. Aspects of performing, collecting, interpreting. Uppsala : KPH Trycksaksbolaget. 


\section{Chanter les territoires Sámi dans un monde plus-qu'humain}

Eriksson J. I. (2002), Samisk shamanism. København:h:ström.

Fagerheim P. (2014), « Sounding Sámi Sentiments. Musical Practices in the Production of Ethnicity », Studia Musicologica Norvegica, vol. 40, p. 63-84.

Gaski H. (2008), "Yoik-Sami Music in a Global World», in Henry MINDE, Indigenous Peoples : Self-Determination, Knowledge, and Indigeneity. Delft : Eburon, p. 347-360.

Gautier A. M. 0. (2016) «Acoustic Multinaturalism, the Value of Nature, and the Nature of Music in Ecomusicology », Boundary 2, vol. 43, no 1, p. 107-141.

Graff 0. (2007), " The relation between Sami Yoik songs and nature », European Meetings in Ethnomusicology, vol. 12, p. 227-231.

Graff 0. (2016), Joikeforbudet i Kautokeino. Karasjok : Davvi Girji.

Guttormsen A. (2014), Avviser at joik er hedensk. Vårt land. En ligne : http://www.vl.no/2.610/avviser-at-joik-er-hedensk-1.28297

Hanssen I. (2011), " A song of identity: Yoik as example of the importance of symbolic cultural expression in intercultural communication/health care », Journal of Intercultural Communication, vol. 27.

Helander E. (1999), "Sami subsistence activities - spatial aspects and structuration », Acta Borealia: A Nordic Journal of Circumpolar Societies, vol. 16, no 2, p. 7-25.

Helander-Renvall E. (2010), « Animism, personhood and the nature of reality: Sami perspectives », Polar Record, vol. 46, no 1, p. 44-56.

Ingold T. (2000), The Perception of the Environment. Essays on livelihood, dwelling and skill. London : Routledge.

Ingold T. (2013), Marcher avec les dragons. Traduit de l'anglais par Pierre Madelin. Bruxelles : Zones Sensibles.

Ingold T. et Kurttila T. (2000), "Perceiving the Environment in Finnish Lapland ». Body and Society, vol. 6, p. 183-196.

Johnston C. (2008), « Beyond the clearing: towards a dwelt animal geography ». Progress in Human Geography, vol. 32, no 5, p. 633-649.

Jones-Bamman R. W. (1993), "As long as we continue to joik, we'll remember who we are. » Negotiating identity and the performance of culture : the saami joik. Washington : University of Washington Press.

Kohn E. (2013), How forests think: toward an anthropology beyond the human. Berkeley : University of California Press.

Latour B. (1991), Nous n'avons jamais été modernes. Essai d'anthropologie symétrique. Paris : La Découverte.

Lüderwaldt A. (1976), Joiken aus Norwegen. Bremen : Übersee-Museum Bremen.

Mazzullo N. et Ingold T. (2008), «Being Along: Place, Time and Movement among Sámi People », in Jørgen Ole Barenholdt et Brynhild Granås, Mobility and Place: enacting northern European peripheries. Aldershot : Ashgate Publishing p. 27-38.

Moseley W. G., Perramond E., Hapke H. M. et Laris P. (2014) An Introduction to HumanEnvironment Geography. Local Dynamics and Global Processes. Chichester : Wiley-Blackwell. 
Retaillé D. (2005), « L'espace mobile », in Pierre Duboscq, Le monde et la centralité. Bordeaux : CNRS - TIDE.

Schanche A. M. (2002), « Den samiske utmarka ». Dieđut, vol. 1, p. 156-170.

Schefferus J. (1678), Histoire de la Laponie, sa description, l'origine, les mœurs, la manière de vivre de ses Habitans, leur Religion, leur Magie, et les choses rares du Païs. Paris : Chez la Veuve Olivier de Varennes.

Smith L. T. (1999), Decolonizing Methodologies. Research and Indigenous Peoples. London : Zed Books.

Somby Á (2007), Joik and the Theory of Knowledge. En ligne : http://alkman1.blogspot.no/2007/07/joik-and-theory-of-knowledge.html.

Stengers I. (2009), Au temps des catastrophes. Résister à la barbarie qui vient. Paris : La Découverte.

Thomassen I. (2010), Hør stammødrenes stemme: En kontekstuell analyse av Mari Boines album Gula gula. Nesna : Høgskolen i Nesna.

Utsi P. (2000), Följ stigen. Guovdageaidnu : DAT.

Viveiros De Castro E. (2009), Métaphysiques cannibales. Lignes d'anthropologie post-structurale. Paris : Presses Universitaires de France.

Watson C. A. (2016), « Ánde Somby Yoiking With The Winged Ones », Releases | Chris Watson. 2016. En ligne : http://chriswatson.net/category/releases/

Weisethaunet H. (2007), "Historiography and complexities: why is music "national" ? », Popular Music History, vol. 2, no 2, p. 169-199.

Weisethaunet H. (2010), « Authenticity Revisited: The Rock Critic and the Changing Real », Popular Music and Society, vol. 33, no 4, p. 465-485.

\section{Sources audio et audio-visuelles}

Boine M. (1989), Gula gula. Real World Records, LP.

Fjellheim F. (2004), Aejlies Gaaltije. Vuelie, LP.

Lundby E. A. (2014), Joikefeber. Ellen Lundby Film \& Media, DVD.

Saari W. (1996), Texas. Rockadillo Records, EP.

Somby Á. (2016), Yoiking with the Winged Ones, Ash International, LP. 